\title{
Florística de trepadeiras em fragmentos de florestas semideciduais em Uberlândia, Minas Gerais, Brasil ${ }^{1}$
}

Floristics of climbing plants in semideciduous forest fragments at Uberlândia, Minas Gerais, Brazil

\author{
Betânia da Cunha Vargas $^{2,3}$ \& Glein Monteiro Araújo ${ }^{2}$
}

\begin{abstract}
Resumo
Trepadeira é um importante componente da composição e riqueza de espécies vegetais em florestas tropicais. Contudo, pesquisas exclusivas com esse grupo ainda são poucas no Brasil. O presente estudo teve como objetivo caracterizar a composição florística e aspectos ecológicos de trepadeiras em três fragmentos de florestas semideciduais em Uberlândia, MG. Para o levantamento florístico foram realizadas coletas mensais de março de 2010 a março de 2011. As espécies foram classificadas quanto ao hábito trepador, modo de ascensão e dispersão. Para calcular a similaridade florística entre as áreas utilizou-se o índice de Jaccard. Nos três fragmentos de floresta semidecidual estudado foram identificadas 99 espécies incluídas em 25 famílias e 62 gêneros. No fragmento da Fazenda São José (FSJ), Fazenda Experimental do Glória (FEG) e Fazenda Irara (FAI) encontrou-se 71, 53 e 52 espécies. As famílias mais ricas em espécies foram Bignoniaceae (16 espécies), Malpighiaceae (12), Sapindaceae (11) e Fabaceae (10). Houve o predomínio de espécies lenhosas e anemocóricas. As espécies dotadas de gavinhas foram as mais numerosas no fragmento da FSJ, enquanto na FEG e na FAI predominaram espécies volúveis. A similaridade florística entre os fragmentos foi menor que 50\% indicando baixa similaridade entre as nossas áreas de estudo.
\end{abstract}

Palavras-chave: forófito, modo de escalada, similaridade florística.

\begin{abstract}
Climbing plants are an important component of the composition and richness of plant species in tropical forests. However, research focused on this group is still rare in Brazil. This paper aimed to characterize the floristic composition and ecological aspects of climbing plants in three fragments of semideciduous forest in Uberlândia, MG. For the floristic surveys plants were collected monthly from March 2010 to March 2011. The species were classified according to climbing habit, climbing strategy and dispersion. To calculate floristic similarity between the fragments we used the Jaccard index. We identified 99 species in 62 genera and 25 families. A total of 71, 53, and 52 species were recorded, respectively, at Fazenda São José (FSJ), Fazenda Experimental do Glória (FEG) and Fazenda Irara (FAI) fragments. The most species-rich families were Bignoniaceae (16 species), Malpighiaceae (12), Sapindaceae (11) and Fabaceae (10). Overall, there was a predominance of woody and anemochoric species. Species with tendrils were most numerous in FSJ, while in FEG and FAI there was a predominance of twining species. Floristic similarity between fragments was less than 50\% indicating low similarity between the study areas. Key words: host tree, climbing strategy, floristic similarity.
\end{abstract}

\section{Introdução}

As trepadeiras são plantas que germinam no solo, permanecem ligadas a este durante todo seu ciclo de vida, e utilizam um suporte mecânico (forófito) para garantir seu crescimento em altura (Gentry 1991; Acevedo-Rodriguez 2005).
Essas plantas mecanicamente dependentes têm despertado atenção dos pesquisadores desde o século XIX, período em que Darwin escreveu um livro onde destacou os movimentos de rotação do caule dessas espécies vegetais (Isnard \& Silk 2009). Diversas denominações foram

\footnotetext{
Dissertação de Mestrado da primeira autora.

${ }^{2}$ Universidade Federal de Uberlândia, Inst. Biologia, Programa de Pós-graduação em Biologia Vegetal, R. Pará s/n, 38400-902, Uberlândia, MG, Brasil.

${ }^{3}$ Autora para correspondência: betaniacunha@yahoo.com.br
} 
relacionadas ao hábito trepador, sendo as mais adotadas aquelas que utilizam as propriedades morfológicas do caule, ou seja, trepadeiras herbáceas e lenhosas (Gentry 1985).

As trepadeiras são consideradas heliófilas, ou seja, são dependentes de luz para o desenvolvimento (Putz 1984) tendo, então, a capacidade de crescer rapidamente em altura e extensão para alcançar dossel (Putz \& Chai 1987). Para isso, desenvolveram mecanismos de ascensão, que são estratégias ecológicas e morfológicas para escalar o forófito e maximizar a captura de luz (Gentry 1985; Hergaty 1991). Assim, a associação trepadeira/forófito é caracterizada pela competição principalmente por recursos como luz, água e nutrientes (Schnitzer 2005). As trepadeiras podem atuar como uma força seletiva na dinâmica da comunidade florestal determinando condições de vida para os demais componentes, atuando assim, como uma verdadeira força seletiva (Putz 1984; Phillips et al. 2005).

Essas plantas são comuns em praticamente qualquer tipo de clima e comunidades vegetais, sendo o diferencial fisionômico entre florestas temperadas e tropicais (Gentry 1991), no qual atingem maior abundância, diversidade e riqueza nos trópicos (Emmons \& Gentry 1983; Schnitzer \& Bongers 2002), onde cerca de 18 a $22 \%$ da diversidade taxonômica são trepadeiras juvenis (Gentry 1991). Lianas contribuem com cerca de $25 \%$ da diversidade nas florestas tropicais (Schnitzer \& Bongers 2002) e na bacia Amazônica essa proporção pode ser de até $44 \%$ (Gentry 1991; Pérez-Salicrup et al. 2001), sendo considerado importante componente florístico, funcional e estrutural das florestas tropicais (Morellato \& Leitão Filho, 1996; Fonseca \& Oliveira 1998).

Estudos realizados com lianas têm demostrado que a abundância e biomassa vem aumentado em florestas tropicais (Phillips et al. 2002), isto relacionado a fatores tais como a sazonalidade da precipitação (DeWalt et al. 2010), topografia do solo (Schnitzer \& Bongers 2002), disponibilidade de estruturas de suporte (Sfair \& Martins 2011), distrúrbios florestais (Laurance et al. 2001; Schnitzer \& Carson 2001) e o aumento de $\mathrm{CO}_{2}$ atmosférico (Granados \& Korner 2002). Sendo que, os meios de produção e uso desordenado da terra têm contribuído para o processo da fragmentação (Tabarelli \& Gascon 2005), que são pequenas manchas florestais com ampla diversidade florística (Klink \& Machado 2005). Nesses fragmentos ocorrem bordas artificiais, que são ambientes bem iluminados que favorecem o desenvolvimento destas plantas (Hergaty \& Caballé 1991; DeWalt et al. 2000).

É notável a importância das trepadeiras nas comunidades vegetais, pois contribuem para diversidade florística, além de promover a conexão das copas das árvores, facilitando assim, o deslocamento de animais não voadores (Putz 1984, 2005). As trepadeiras também fornecem recursos alimentares, principalmente em época de baixa disponibilidade da comunidade arbórea (Morellato \& Leitão Filho 1996) e auxiliam na manutenção do micro clima durante a estação seca e fria (Schnitzer \& Bongers 2002).

Apesar da importância e ampla distribuição das trepadeiras, estas receberam menos enfoque em estudos nas florestas tropicais em relação a outras formas de vida (Gentry 1991; Udulutsch et al. 2010). Essa carência de informações pode estar relacionada às dificuldades de coleta no dossel da floresta (Putz 1984; Villagra \& Neto 2010) e também estar associado a problemas na identificação desse grupo tão diverso (Udulutsch et al. 2010).

No Brasil, os estudos com florística de trepadeiras datam da década de 90 , sendo que grande parte destes foram realizados nas florestas semideciduais do estado de São Paulo. Estes estudos constituíram-se de levantamentos florísticos (Bernacci \& Leitão Filho 1996; Morellato \& Leitão Filho 1996, 1998; Udulutsch et al. 2004; Rezende \& Ranga 2005; Tibiriçá et al. 2006), amostragens fitossociológicas (Hora \& Soares 2002; Rezende et al. 2007) e investigações sobre a relação entre lianas e a diversidade arbórea (Sfair \& Martins 2011). O modo de ascensão foi motivo de pesquisa em alguns dos estudos sobre trepadeiras (Tibiriçá et al 2006; Santos et al. 2009; Villagra \& Neto 2010), sendo outros aspectos ecológicos, como hábito e formas de dispersão, ainda pouco explorados (Morellato \& Leitão Filho 1998). O estudo de Santos et al. (2009) reuniu diversos trabalhos com trepadeiras de florestas semideciduais e buscou identificar padrões para esta forma de vida.

Nesse contexto, o objetivo deste trabalho foi caracterizar a comunidade de trepadeiras em três fragmentos de florestas semideciduais no município de Uberlândia, visando classificar as espécies quanto ao hábito trepador, modo de ascensão, formas de dispersão e ocorrência nos fragmentos. 


\section{Material e Métodos}

O estudo das trepadeiras foi realizado em três fragmentos de florestas semideciduais localizados em reservas legais na Fazenda Experimental do Glória (FEG), Fazenda São José (FSJ) e Fazenda Irara (FAI) todas no município de Uberlândia, Minas Gerais (Fig. 1). Os três fragmentos possuem em seu interior áreas com nascentes de córregos da bacia do rio Araguari e estão ligadas às florestas de galeria que margeiam esses cursos de água. Além disso, estão inseridos em uma matriz com lavouras, áreas de pastagens e reflorestamento com eucalipto. Os fragmentos são bem preservados, com poucas clareiras internas, mas as bordas possuem alguns trechos com densa cobertura de trepadeiras.

O fragmento de floresta semidecidual da FEG $\left(48^{\circ} 12^{\prime} 22^{\prime \prime} \mathrm{W}\right.$ e $\left.18^{\circ} 57^{\prime} 03^{\prime \prime} \mathrm{S}\right)$ pertence à Universidade Federal de Uberlândia (UFU) e possui cerca de 30 ha. O solo sob a floresta é do tipo Latossolo Vermelho-Escuro distrófico com textura argilosa possuí teor médio de $\mathrm{Ca}^{+2}$ disponível de 1,81 $\mathrm{Cmolc} \mathrm{dm}^{-3}$ e saturação média de bases de $16,8 \%$ (Haridasan \& Araújo 2005). O fragmento de floresta semidecidual $\left(48^{\circ} 08^{\prime} 46^{\prime \prime} \mathrm{W}\right.$ e $\left.19^{\circ} 08^{\prime} 39^{\prime \prime} \mathrm{S}\right)$ situado na FAI tem cerca de 20 ha. O solo sob este fragmento é distrófico, com teor médio de

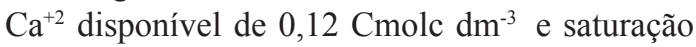

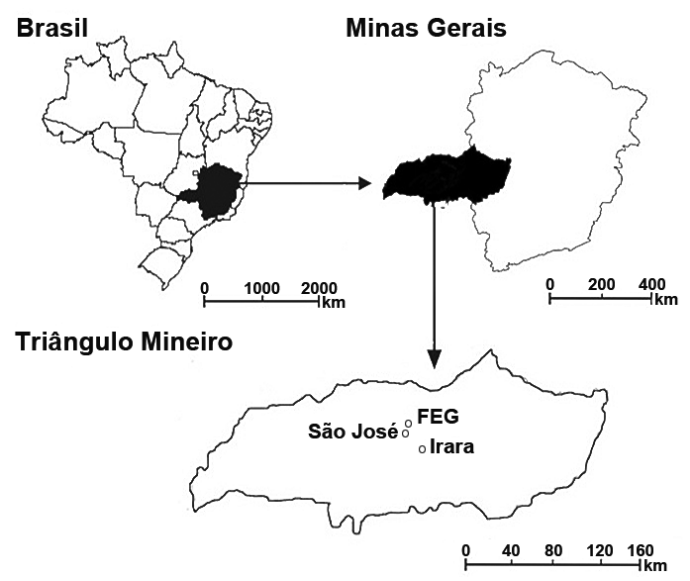

Figura 1 - Localização dos fragmentos de floresta semidecidual dentro da região do Triângulo Mineiro e Alto Paranaíba, MG. FEG = Fazenda Experimental do Glória; FSJ = Fazenda São José; FAI = Fazenda Irara. Adaptado de Lopes et al. (2012).

Figure 1 - Location of the fragments of semideciduous forest within the Triângulo Mineiro e Alto Paranaíba region. Fazenda Experimental do Glória; FSJ = Fazenda São José; FAI = Fazenda Irara. Adapted from Lopes et al. (2012). de bases de 2,6\% (Lopes 2010). O fragmento de floresta semidecidual ( $48^{\circ} 13^{\prime} 53^{\prime \prime} \mathrm{W}$ e $18^{\circ} 51^{\prime} 35^{\prime}$ 'S) da FSJ possuiu cerca de 20 ha e tem o solo diferente dos anteriores, com setores de fertilidades bem contrastantes. No setor Noroeste e Norte foram registrados teor médio de $\mathrm{Ca}^{+2}$ disponível de 2,68 Cmolc dm ${ }^{-3}$ e saturação de bases de $40,17 \%$ tendo cerca de 10 vezes maior fertilidade do que o setor Leste e Sul com de $\mathrm{Ca}^{+2}$ disponível de 0,16 Cmolc $\mathrm{dm}^{-3} \mathrm{e}$ saturação de bases de 4,11\% (Lopes 2010).

O clima predominante da região, segundo a classificação de Köppen (1948), é do tipo Tropical Savana (Aw). É um clima sazonal caracterizado por um período chuvoso no verão, de outubro a março e relativamente seco no inverno, de abril a setembro. A temperatura média anual é de $22^{\circ} \mathrm{C}$, com total pluviométrico de $1500 \mathrm{~mm} /$ ano (Silva et al. 2008).

O levantamento florístico das trepadeiras nos três fragmentos de floresta semidecidual foi realizado mensalmente, no período de março de 2010 a março de 2011. Foram percorridas as bordas dos fragmentos coletando-se as espécies em estágio reprodutivo. As coletas foram realizadas na borda devido à dificuldade de visualizar e coletar as trepadeiras no interior dos fragmentos que possuem árvores altas com densa cobertura de copa. Os exemplares coletados foram herborizados e identificados por meio de chaves analíticas e descrições bibliográficas específicas. Também foram feitas comparações com material depositado no Herbarium Uberlandense (HUFU) e herbários da Universidade Estadual de Campinas (UEC), Herbário Escola Superior de Agricultura Luiz de Queiroz (ESA), além de consultas a especialistas. O sistema de classificação para as famílias foi a APG III (Bremer et al. 2009). A grafia e as identificações das espécies foram padronizadas de acordo com a Lista da flora brasileira de 2012 disponível no site $<$ http://floradobrasil.jbrj.gov. br/2012/>, administrada pelo Jardim Botânico do Rio de Janeiro. O material coletado nos fragmentos florestais foi incorporado ao acervo do Herbarium Uberlandense da Universidade Federal de Uberlândia, tendo duplicatas nos herbários visitados.

As espécies de trepadeiras coletadas foram classificadas segundo o hábito trepador, de acordo com Gentry (1991), em herbáceas e lenhosas. Pra a classificação quanto ao modo de escalada, adotaram-se as quatro categorias (Hergaty 1991): volúveis, as que envolvem o forófito com movimento helicoidal por meio do caule 
principal, ramos, pecíolos e peciólulos; gavinha, que possuem estruturas caulinares, foliares e outras modificadas em gavinha; apoiantes, que se apóiam passivamente, podendo apresentar ganchos ou espinhos que auxiliam o apoio no forófito e radicantes, aquelas que usam raízes adventícias para fixarem no suporte.

Para cada espécie coletada foi determinada a síndrome de dispersão, utilizando-se as denominações usuais: anemocóricas com dispersão pelo vento; zoocóricas com dispersão por animais e autocóricas quando as características do sistema de dispersão não se enquadram nas categorias anteriores e tinham características de autodispersão (Van Der Pijil 1982; Morellato \& Leitão Filho 1998).

A similaridade florística entre os três fragmentos florestais foi calculada utilizandose o índice de Jaccard (Mueller-Dombois \& Ellenberg 1974).

Para verificar se os modos de ascensão (volúvel, apoiante e gavínea) diferem entre os três fragmentos florestais utilizou-se o teste do Qui quadrado. As análises foram feitas no programa Systat.

\section{Resultados}

O número total de espécies de trepadeiras encontradas nos três fragmentos florestais foi de 99 espécies distribuídas em 26 famílias e 63 gêneros. A maior riqueza ocorreu no fragmento da FSJ com 71 espécies seguidas dos fragmentos das FEG e FAI com 53 e 52 espécies (Tabs. 1, 2). O maior número de espécies exclusivas e compartilhadas ocorreu no fragmento da FSJ (Fig. 2).

As famílias com maior riqueza de espécies foram Bignoniaceae (16 espécies), Malpighiaceae (12), Sapindaceae (11), Fabaceae (10), Apocynaceae (9), Convolvulaceae e Asteraceae (5), tendo juntas $70 \%$ das espécies amostradas. No entanto, a ordem de riqueza específica das famílias variou entre os fragmentos. As famílias Amaranthaceae e Canabaceae foram amostradas apenas no fragmento da FSJ, e Dioscoreaceae e Polygonaceae apenas no fragmento da FAI enquanto no fragmento da FEG não foi amostrado nenhuma família exclusiva (Tab. 1).

Em relação ao hábito registrou-se, em cada fragmento, mais de $70 \%$ de trepadeiras lenhosas. Bignoniaceae, Malpighiaceae e Sapindaceae, famílias exclusivamente lenhosas, foram as mais ricas em espécies (Tab. 1).

Nos três fragmentos, o modo de ascensão volúvel foi o mais encontrado (44 espécies), seguido daquelas dotadas de gavinha (37) e por fim as apoiantes (18). No entanto, no fragmentos FSJ gavinha foi o tipo de escalada com o maior número de espécies (Tab. 2). Os modos de ascensão não diferiram entre os três fragmentos florestais estudados $\mathrm{c}^{2}=2,45, \mathrm{gl}=4, \mathrm{p}<0,05$. As famílias Bignoniaceae e Sapindaceae tiveram espécies exclusivamente com gavinhas, enquanto Apocynaceae, Convolvulaceae e Malpighiaceae mostraram-se espécies unicamente com modo de ascensão volúvel. Outras famílias como Malpighiaceae, Fabaceae e Asteraceae tiveram mais de um modo de ascensão (Tab. 1). Registrouse também, no fragmento da FAI, a espécie Dolichandra ungüis-cati que durante uma fase do seu desenvolvimento apresentou ascensão por meio de raiz adventícia.

A anemocoria foi o sistema de dispersão encontrado em maior número de espécies (67 espécies) nas três florestas amostradas, as trepadeiras herbáceas tiveram maior número de espécies zoocóricas (11) enquanto as lenhosas mais espécies anemocóricas (60).

A similaridade florística entre os três fragmentos florestais variou de $32,5 \%$ a $42,1 \%$ sendo a maior entre a Fazenda Experimental do Glória e a Fazenda São José (Tab. 3).

\section{Discussão}

Nos três fragmentos florestais inventariados neste estudo encontraram-se 2 a 3 vezes menos espécies de trepadeiras do que em florestas semideciduais paulistas estudadas por Morelato \& Leitão Filho (1998), Udulutsch et al. (2004), Tibiriçá et al. (2006). O maior tamanho dos fragmentos florestais paulistas, o método e esforço amostral (aqui se amostrou apenas a borda do fragmento) e histórico de perturbação como relatados por Morelato \& Leitão Filho (1996), Tibiriçá et al. (2006), Udulutsch et al. (2010) podem ter interferido nessa diferença.

Apesar dos três fragmentos florestais situarem-se em um mesmo município, ter aproximadamente o mesmo tamanho e histórico de perturbação o fragmento da FSJ teve maior número de espécies do que os demais fragmentos aqui inventariados. A heterogeneidade do fragmento florestal (Tibiriçá et al. 2006; Udulutsch et al. 2004) como diferenças internas nas características do solo, como foi encontrada na floresta da FSJ por Lopes (2010) pode ter interferido no maior número de trepadeiras amostradas. 
Tabela 1 - Espécies de trepadeiras amostradas nas florestas semideciduais do município de Uberlândia, MG. Locais de ocorrência: FSJ = Fazenda São José; FEG = Fazenda Experimental do Glória; FAI = Fazenda Irara. Hábito: H = herbacea; $\mathrm{L}=$ lenhosa. Modo de ascensao: $\mathrm{Vol}=$ Voluvel; Gav= gavinha; Apo= apoiante; RAd.= raiz adventicia. Ocorrência: $\mathrm{R}=$ rara; $\mathrm{O}=$ ocasional; $\mathrm{Ab}=$ abundante. Dispersão: $\mathrm{A}=$ =anemocórica; $\mathrm{Au}=$ autocórica; $\mathrm{Z}=$ zoocórica. HUFU: Número de registro das exsicatas depositadas no Herbarium Uberlandense.

Table 1 - Climbing species sampled in fragments of semideciduous forests in the city of Uberlândia, MG, Brazil. Places of occurrence: FSJ = Fazenda São José; FEG = Fazenda Experimental do Glória; FAI = Fazenda Irara. Habit: H = herbaceous ; L = woody. Climbing strategy: Vol = twining; $\mathrm{Gav}=$ tendril climbing; $\mathrm{Apo}=$ scramblers; $\mathrm{Rad}=$ Adventitious root. Occurrence: $\mathrm{R}=$ rare; $\mathrm{O}=$ occasional; $\mathrm{Ab}=$ abundant. Dispersal mode: $\mathrm{A}=$ wind-dispersed; $\mathrm{Au}=$ self-dispersed; $\mathrm{Z}=$ animal-dispersed. HUFU: Voucher number of specimens deposited at the Herbarium Uberlandense.

\begin{tabular}{|c|c|c|c|c|c|c|c|}
\hline \multirow{2}{*}{ Família/Espécie } & \multicolumn{3}{|c|}{ Ocorrência } & \multirow{2}{*}{ Hábito } & \multirow{2}{*}{ Ascensão } & \multirow{2}{*}{ Dispersão } & \multirow{2}{*}{ HUFU } \\
\hline & FSJ & FEG & FAI & & & & \\
\hline \multicolumn{8}{|l|}{ Acanthaceae } \\
\hline Justicia sp. & $\mathrm{R}$ & - & - & $\mathrm{H}$ & Apo & A & 57.559 \\
\hline \multicolumn{8}{|l|}{ Amaranthaceae } \\
\hline Froelichia lanada Moench & $\mathrm{R}$ & - & - & $\mathrm{L}$ & Apo & A & 58.729 \\
\hline \multicolumn{8}{|l|}{ Apocynaceae } \\
\hline Condylocarpon isthmicum (Vell.) A.DC. & $\mathrm{R}$ & - & $\mathrm{R}$ & $\mathrm{L}$ & Vol & A & 58.901 \\
\hline Ditassa burchellii Hook. \& Arn. & $\mathrm{R}$ & - & - & $\mathrm{L}$ & Vol & A & 60.046 \\
\hline Forsteronia pubescens A.DC. & $\mathrm{R}$ & - & $\mathrm{R}$ & $\mathrm{L}$ & Vol & A & 58.863 \\
\hline Mandevilla hirsuta (A.Rich.) K.Schum. & - & - & $\mathrm{R}$ & $\mathrm{H}$ & Vol & A & 58.825 \\
\hline Oxypetalum erianthum Decne. & $\mathrm{R}$ & - & - & $\mathrm{H}$ & Vol & A & 57.048 \\
\hline Prestonia coalita (Vell.) Woodson & $\mathrm{R}$ & - & $\mathrm{O}$ & $\mathrm{L}$ & Vol & A & 56.955 \\
\hline Prestonia riedelii (Müll.Arg.) Markgr. & $\mathrm{R}$ & $\mathrm{O}$ & $\mathrm{R}$ & $\mathrm{L}$ & Vol & A & 59.089 \\
\hline Schubertia grandiflora Mart. & - & $\mathrm{Ab}$ & - & $\mathrm{H}$ & Vol & A & 59.353 \\
\hline Secondatia densiflora A.DC. & $\mathrm{R}$ & - & $\mathrm{R}$ & $\mathrm{L}$ & Vol & A & 58.870 \\
\hline \multicolumn{8}{|l|}{ Aristolochiaceae } \\
\hline Aristolochia labiata Willd. & - & - & $\mathrm{R}$ & $\mathrm{L}$ & Vol & A & 58.864 \\
\hline Aristolochia melastoma Silva Manso ex Duch. & $\mathrm{R}$ & - & - & $\mathrm{L}$ & Vol & A & 59.349 \\
\hline \multicolumn{8}{|l|}{ Asteraceae } \\
\hline Bidens segetum Mart. ex Colla & $\mathrm{O}$ & $\mathrm{O}$ & - & $\mathrm{L}$ & Vol & A & 57.056 \\
\hline Dasyphyllum flagellare (Casar.) Cabrera & $\mathrm{O}$ & - & $\mathrm{O}$ & $\mathrm{L}$ & Apo & A & 56.912 \\
\hline Mikania cordifolia (L.f.) Willd. & $\mathrm{R}$ & - & - & $\mathrm{H}$ & Vol & A & 57.566 \\
\hline Mikania micrantha Kunth & $\mathrm{R}$ & - & - & $\mathrm{H}$ & Vol & A & 47.963 \\
\hline Mikania psilostachya DC. & $\mathrm{R}$ & $\mathrm{R}$ & - & $\mathrm{L}$ & Apo & A & 56.937 \\
\hline \multicolumn{8}{|l|}{ Bignoniaceae } \\
\hline Adenocalymma bracteatum (Cham.) DC & $\mathrm{R}$ & - & - & $\mathrm{L}$ & Gav & A & 57.110 \\
\hline Amphilophium elongatum (Vahl) L.G.Lohmann & $\mathrm{O}$ & $\mathrm{R}$ & $\mathrm{R}$ & $\mathrm{L}$ & Gav & A & 58.833 \\
\hline Amphilophium paniculatum (L.) Kunth & $\mathrm{R}$ & - & - & $\mathrm{L}$ & Gav & A & 59.083 \\
\hline Cuspidaria pulchra (Cham.) L.G.Lohmann & $\mathrm{Ab}$ & $\mathrm{Ab}$ & $\mathrm{Ab}$ & $\mathrm{L}$ & Gav & A & 57.583 \\
\hline Dolichandra unguis-cati (L.) L.G.Lohmann & - & - & $\mathrm{R}$ & $\mathrm{L}$ & Gav/RAd. & A & 58.839 \\
\hline Fridericia candicans (Rich.) L.G.Lohmann & $\mathrm{O}$ & $\mathrm{Ab}$ & $\mathrm{O}$ & $\mathrm{L}$ & Gav & A & 59.086 \\
\hline Fridericia craterophora (DC.) L.G.Lohmann & - & $\mathrm{R}$ & - & $\mathrm{L}$ & Gav & A & 56.930 \\
\hline Fridericia florida (DC.) L.G.Lohmann & $\mathrm{Ab}$ & $\mathrm{Ab}$ & $\mathrm{Ab}$ & $\mathrm{L}$ & Gav & A & 56.900 \\
\hline Fridericia formosa (Bureau) L.G.Lohmann & $\mathrm{O}$ & - & $\mathrm{O}$ & $\mathrm{L}$ & Gav & A & 57.113 \\
\hline
\end{tabular}




\begin{tabular}{|c|c|c|c|c|c|c|c|}
\hline \multirow{2}{*}{ Família/Espécie } & \multicolumn{3}{|c|}{ Ocorrência } & \multirow{2}{*}{ Hábito } & \multirow{2}{*}{ Ascensão } & \multirow{2}{*}{ Dispersão } & \multirow{2}{*}{ HUFU } \\
\hline & S.J. & FEG & Ira & & & & \\
\hline Fridericia speciosa Mart. & - & $\mathrm{O}$ & $\mathrm{O}$ & $\mathrm{L}$ & Gav & A & 56.939 \\
\hline Fridericia triplinervia (Mart. ex DC.) L.G.Lohmann & $\mathrm{O}$ & - & - & $\mathrm{L}$ & Gav & A & 56.905 \\
\hline Lundia cordata (Vell.) DC. & $\mathrm{R}$ & - & - & $\mathrm{L}$ & Gav & A & 56.914 \\
\hline Pyrostegia venusta (Ker Gawl.) Miers & $\mathrm{Ab}$ & $\mathrm{Ab}$ & $\mathrm{Ab}$ & $\mathrm{L}$ & Gav & A & 57.581 \\
\hline Stizophyllum perforatum (Cham.) Miers & - & $\mathrm{R}$ & - & $\mathrm{L}$ & Gav & A & 58.837 \\
\hline Stizophyllum riparium (Kunth) Sandwith & $\mathrm{O}$ & $\mathrm{O}$ & - & $\mathrm{L}$ & Gav & A & 57.569 \\
\hline Tanaecium pyramidatum (Rich.) L.G.Lohmann & $\mathrm{O}$ & $\mathrm{O}$ & $\mathrm{O}$ & $\mathrm{L}$ & Gav & A & 58.796 \\
\hline \multicolumn{8}{|l|}{ Cannabaceae } \\
\hline Celtis iguanaea (Jacq.) Sarg. & $\mathrm{O}$ & - & - & $\mathrm{L}$ & Apo & $\mathrm{Z}$ & 58.809 \\
\hline \multicolumn{8}{|l|}{ Celastraceae } \\
\hline Pristimera celastroides (Kunth) A.C.Sm & $\mathrm{R}$ & - & $\mathrm{R}$ & $\mathrm{L}$ & Apo & $\mathrm{Z}$ & 58.877 \\
\hline \multicolumn{8}{|l|}{ Convolvulaceae } \\
\hline Bonamia burchellii (Choisy) Hallier f. & $\mathrm{R}$ & $\mathrm{R}$ & - & $\mathrm{H}$ & Vol & A & 56.899 \\
\hline Ipomoea saopaulista O’Donell & - & $\mathrm{R}$ & - & $\mathrm{H}$ & Vol & $\mathrm{Au}$ & 56.933 \\
\hline Ipomoea tubata Nees & $\mathrm{R}$ & $\mathrm{R}$ & - & $\mathrm{H}$ & Vol & $\mathrm{Au}$ & 57.111 \\
\hline Jacquemontia ferruginea Choisy & - & - & $\mathrm{R}$ & $\mathrm{H}$ & Vol & $\mathrm{Au}$ & 58.798 \\
\hline Merremia macrocalyx (Ruiz \& Pav.) O’Donell & $\mathrm{O}$ & $\mathrm{O}$ & - & $\mathrm{H}$ & Vol & $\mathrm{Au}$ & 56.941 \\
\hline \multicolumn{8}{|l|}{ Cucurbitaceae } \\
\hline Gurania lobata (L.) Pruski & - & - & $\mathrm{R}$ & $\mathrm{H}$ & Gav & $\mathrm{Z}$ & 58.869 \\
\hline Psiguria ternata (M.Roem.) C.Jeffrey & - & - & $\mathrm{R}$ & $\mathrm{H}$ & Gav & $\mathrm{Z}$ & 59.342 \\
\hline \multicolumn{8}{|l|}{ Dilleniaceae } \\
\hline Doliocarpus dentatus (Aubl.) Standl. & $\mathrm{R}$ & $\mathrm{R}$ & - & $\mathrm{L}$ & Vol & $\mathrm{Z}$ & 57.558 \\
\hline \multicolumn{8}{|l|}{ Dioscoreaceae } \\
\hline Dioscorea glandulosa (Griseb.) Kunth & - & - & $\mathrm{R}$ & $\mathrm{H}$ & Vol & $\mathrm{Z}$ & 57.036 \\
\hline Dioscorea multiflora Mart. ex Griseb & - & - & $\mathrm{R}$ & $\mathrm{H}$ & Vol & $\mathrm{Z}$ & 57.574 \\
\hline \multicolumn{8}{|l|}{ Fabaceae } \\
\hline Camptosema ellipticum (Desv.) Burkart & - & - & $\mathrm{R}$ & $\mathrm{H}$ & Vol & $\mathrm{Au}$ & 60.056 \\
\hline Canavalia brasiliensis Mart. ex Benth. & - & $\mathrm{R}$ & - & $\mathrm{H}$ & Vol & $\mathrm{Au}$ & 57.025 \\
\hline Clitoria laurifolia Poir. & - & $\mathrm{O}$ & - & $\mathrm{H}$ & Vol & $\mathrm{Au}$ & 60.055 \\
\hline Clitoria ternatea $\mathrm{L}$. & - & $\mathrm{R}$ & $\mathrm{R}$ & $\mathrm{H}$ & Vol & $\mathrm{Au}$ & 60.136 \\
\hline Dioclea bicolor Benth. & - & $\mathrm{O}$ & - & $\mathrm{L}$ & Vol & $\mathrm{Au}$ & 57.503 \\
\hline Dioclea violacea Mart. ex Benth. & $\mathrm{R}$ & - & $\mathrm{R}$ & $\mathrm{L}$ & Vol & $\mathrm{Au}$ & 57.551 \\
\hline Machaerium hirtum (Vell.) Stellfeld & $\mathrm{R}$ & $\mathrm{R}$ & - & $\mathrm{L}$ & Apo & $\mathrm{A}$ & 57.506 \\
\hline Rhynchosia melanocarpa Grear & $\mathrm{R}$ & - & $\mathrm{R}$ & $\mathrm{H}$ & Vol & $\mathrm{Z}$ & 57.121 \\
\hline Senna sp. & $\mathrm{R}$ & - & - & $\mathrm{L}$ & Apo & A & 57.123 \\
\hline Senna splendida (Vogel) H.S.Irwin \& Barneby & $\mathrm{R}$ & - & $\mathrm{R}$ & $\mathrm{L}$ & Apo & A & 57.045 \\
\hline \multicolumn{8}{|l|}{ Malpighiaceae } \\
\hline Banisteriopsis adenopoda (A.Juss.) B.Gates & $\mathrm{O}$ & $\mathrm{O}$ & $\mathrm{O}$ & $\mathrm{L}$ & Vol & A & 57.592 \\
\hline Banisteriopsis argyrophylla (A.Juss.) B.Gates & $\mathrm{Ab}$ & $\mathrm{Ab}$ & $\mathrm{Ab}$ & $\mathrm{L}$ & Vol & A & 57.028 \\
\hline Banisteriopsis laevifolia (A.Juss.) B.Gates & $\mathrm{O}$ & $\mathrm{O}$ & $\mathrm{O}$ & $\mathrm{L}$ & Apo & A & 58.823 \\
\hline Banisteriopsis malifolia (Nees \& Mart.) B.Gates & - & - & $\mathrm{R}$ & $\mathrm{L}$ & Vol & A & 56.949 \\
\hline Banisteriopsis oxyclada (A.Juss.) B.Gates & $\mathrm{O}$ & $\mathrm{O}$ & - & $\mathrm{L}$ & Vol & A & 57.109 \\
\hline
\end{tabular}




\begin{tabular}{|c|c|c|c|c|c|c|c|}
\hline \multirow{2}{*}{ Família/Espécie } & \multicolumn{3}{|c|}{ Ocorrência } & \multirow{2}{*}{ Hábito } & \multirow{2}{*}{ Ascensão } & \multirow{2}{*}{ Dispersão } & \multirow{2}{*}{ HUFU } \\
\hline & S.J. & FEG & Ira & & & & \\
\hline Carolus sp. & $\mathrm{R}$ & $\mathrm{R}$ & - & $\mathrm{L}$ & Vol & A & 57.044 \\
\hline Diplopterys lutea (Griseb.) W.R.Anderson \& C.C.Davis & $\mathrm{R}$ & $\mathrm{R}$ & - & $\mathrm{L}$ & Vol & A & 58.784 \\
\hline Diplopterys pubipetala (A.Juss.) W.R.Anderson \& C.C.Davis & $\mathrm{O}$ & $\mathrm{O}$ & $\mathrm{O}$ & $\mathrm{L}$ & Vol & A & 57.553 \\
\hline Heteropterys anoptera A.Juss. & $\mathrm{R}$ & $\mathrm{R}$ & - & $\mathrm{L}$ & Vol & A & 57.557 \\
\hline Heteropterys campestris A.Juss. & - & $\mathrm{O}$ & $\mathrm{R}$ & $\mathrm{L}$ & Apo & A & 57.593 \\
\hline Heteropterys cochleosperma A.Juss. & - & $\mathrm{R}$ & - & $\mathrm{L}$ & Vol & A & 56.945 \\
\hline Mascagnia cordifolia (A.Juss.) Griseb. & $\mathrm{R}$ & $\mathrm{R}$ & $\mathrm{R}$ & $\mathrm{L}$ & Vol & A & 58.805 \\
\hline \multicolumn{8}{|l|}{ Menispermaceae } \\
\hline Cissampelos glaberrima A.St.-Hil. & - & $\mathrm{Ab}$ & $\mathrm{Ab}$ & $\mathrm{L}$ & Vol & Z & 60.041 \\
\hline Odontocarya acuparata Miers & $\mathrm{R}$ & $\mathrm{R}$ & $\mathrm{R}$ & $\mathrm{L}$ & Vol & $\mathrm{Z}$ & 58.895 \\
\hline \multicolumn{8}{|l|}{ Passifloraceae } \\
\hline Passiflora amethystina J.C.Mikan & $\mathrm{R}$ & $\mathrm{R}$ & - & $\mathrm{H}$ & Gav & $\mathrm{Z}$ & 57.176 \\
\hline Passiflora gibertii N.E.Br. & $\mathrm{R}$ & - & - & $\mathrm{H}$ & Gav & $\mathrm{Z}$ & 57.546 \\
\hline Passiflora miersii Mast. & $\mathrm{R}$ & - & $\mathrm{R}$ & $\mathrm{H}$ & Gav & $\mathrm{Z}$ & 57.390 \\
\hline Passiflora tricuspis Mast. & $\mathrm{Ab}$ & $\mathrm{R}$ & $\mathrm{Ab}$ & $\mathrm{H}$ & Gav & $\mathrm{Z}$ & 57.119 \\
\hline \multicolumn{8}{|l|}{ Polygalaceae } \\
\hline Bredemeyera floribunda Willd. & - & $\mathrm{O}$ & $\mathrm{O}$ & $\mathrm{L}$ & Apo & A & 57.577 \\
\hline Securidaca tomentosa A.St.-Hil. \& Moq. & $\mathrm{R}$ & - & - & $\mathrm{L}$ & Apo & A & 56.915 \\
\hline Securidaca rivinifolia A.St.-Hil. \& Moq. & - & $\mathrm{R}$ & - & $\mathrm{L}$ & Apo & A & 57.505 \\
\hline \multicolumn{8}{|l|}{ Polygonaceae } \\
\hline Coccoloba sp. & - & - & $\mathrm{R}$ & $\mathrm{L}$ & Vol & $\mathrm{Z}$ & 57.389 \\
\hline \multicolumn{8}{|l|}{ Ranunculaceae } \\
\hline Clematis dioica $\mathrm{L}$. & $\mathrm{O}$ & $\mathrm{Ab}$ & - & $\mathrm{L}$ & Vol & A & 57.120 \\
\hline \multicolumn{8}{|l|}{ Rosaceae } \\
\hline Rubus sellowii Cham. \& Schltdl. & - & $\mathrm{R}$ & $\mathrm{R}$ & $\mathrm{L}$ & Apo & $\mathrm{Z}$ & 57.385 \\
\hline \multicolumn{8}{|l|}{ Rubiaceae } \\
\hline Chomelia ribesioides Benth. ex A. Gray & $\mathrm{O}$ & - & - & $\mathrm{L}$ & Apo & $\mathrm{Z}$ & 56.908 \\
\hline \multicolumn{8}{|l|}{ Sapidanceae } \\
\hline Cardiospermum grandiflorum Sw. & $\mathrm{O}$ & - & - & $\mathrm{L}$ & Gav & A & 56.916 \\
\hline Paullinia meliifolia Juss. & $\mathrm{R}$ & $\mathrm{R}$ & - & $\mathrm{L}$ & Gav & A & 58.812 \\
\hline Serjania caracasana (Jacq.) Willd. & $\mathrm{O}$ & - & - & $\mathrm{L}$ & Gav & A & 57.560 \\
\hline Serjania glutinosa Radlk. & $\mathrm{O}$ & - & $\mathrm{O}$ & $\mathrm{L}$ & Gav & A & 57.033 \\
\hline Serjania laruotteana Cambess. & $\mathrm{R}$ & $\mathrm{R}$ & - & $\mathrm{L}$ & Gav & A & 57.049 \\
\hline Serjania lethalis A.St.-Hil. & $\mathrm{R}$ & $\mathrm{O}$ & - & $\mathrm{L}$ & Gav & A & 58.782 \\
\hline Serjania mansiana Mart. & $\mathrm{R}$ & $\mathrm{Ab}$ & $\mathrm{R}$ & $\mathrm{L}$ & Gav & A & 57.030 \\
\hline Serjania meridionalis Cambess. & $\mathrm{O}$ & - & $\mathrm{O}$ & $\mathrm{L}$ & Gav & A & 56.907 \\
\hline Serjania paradoxa Radlk. & $\mathrm{Ab}$ & $\mathrm{Ab}$ & $\mathrm{Ab}$ & $\mathrm{L}$ & Gav & A & 57.058 \\
\hline Serjania pinnatifolia Radlk. & $\mathrm{R}$ & - & - & $\mathrm{L}$ & Gav & A & 57.047 \\
\hline Serjania reticulata Cambess. & $\mathrm{R}$ & $\mathrm{R}$ & $\mathrm{R}$ & $\mathrm{L}$ & Gav & A & 57.562 \\
\hline \multicolumn{8}{|l|}{ Smilacaceae } \\
\hline Smilax brasiliensis Spreng. & $\mathrm{R}$ & $\mathrm{R}$ & $\mathrm{R}$ & $\mathrm{L}$ & Gav & $\mathrm{Z}$ & 58.894 \\
\hline Smilax fluminensis Steud. & $\mathrm{R}$ & - & $\mathrm{R}$ & $\mathrm{L}$ & Gav & $\mathrm{Z}$ & 58.861 \\
\hline
\end{tabular}




\begin{tabular}{|c|c|c|c|c|c|c|c|}
\hline \multirow{2}{*}{ Família/Espécie } & \multicolumn{3}{|c|}{ Ocorrência } & \multirow{2}{*}{ Hábito } & \multirow{2}{*}{ Ascensão } & \multirow{2}{*}{ Dispersão } & \multirow{2}{*}{ HUFU } \\
\hline & S.J. & FEG & Ira & & & & \\
\hline \multicolumn{8}{|l|}{ Verbenaceae } \\
\hline Lantana cujabensis Schauer & - & $\mathrm{R}$ & $\mathrm{R}$ & $\mathrm{L}$ & Apo & Z & 56.951 \\
\hline Petrea volubilis L. & - & - & $\mathrm{R}$ & $\mathrm{L}$ & Apo & A & 58.802 \\
\hline \multicolumn{8}{|l|}{ Violaceae } \\
\hline Anchietea pyrifolia (Mart.) G.Don & $\mathrm{R}$ & $\mathrm{O}$ & - & $\mathrm{L}$ & Vol & A & 57.567 \\
\hline \multicolumn{8}{|l|}{ Vitaceae } \\
\hline Cissus erosa Rich. & $\mathrm{Ab}$ & $\mathrm{Ab}$ & $\mathrm{Ab}$ & $\mathrm{H}$ & Gav & Z & 56.903 \\
\hline Cissus verticillata (L.) Nicolson \& C.E.Jarvis subsp. verticillata & $\mathrm{R}$ & - & - & $\mathrm{H}$ & Gav & $\mathrm{Z}$ & 56.902 \\
\hline
\end{tabular}

Tabela 2 - Aspectos florísticos e ecológicos das trepadeiras amostradas nas florestas semideciduais em Uberlândia, MG. Fragmento florestal: FSJ= Fazenda São José; FEG= Fazenda Experimental do Glória; FAI= Fazenda Irara.

Table 2 - Floristic and ecological aspects of the climbing plants sampled in semideciduous forests in Uberlândia, MG, Brazil. Forest fragment: FSJ= Fazenda São José; FEG= Fazenda Experimental do Glória; FAI= Fazenda Irara.

\begin{tabular}{lcccc}
\hline \multirow{2}{*}{ Características } & \multicolumn{3}{c}{ Fragmento } \\
\cline { 3 - 5 } & & FSJ & FEG & FAI \\
\hline \multirow{2}{*}{ Riqueza } & Espécies & 71 & 53 & 52 \\
& Famílias & 21 & 17 & 19 \\
Hábito (\%) & Herbáceo & 18,3 & 21 & 25 \\
& Lenhoso & 81,7 & 79 & 75 \\
Dispersão (\%) & Anemocórica & 76,1 & 69,8 & 61,5 \\
& Zoocórica & 19,7 & 17 & 30,8 \\
Modo de ascensão (\%) & Autocórica & 4,2 & 13,2 & 7,7 \\
& Volúvel & 39,4 & 47,2 & 42,3 \\
& Apoiante & 16,9 & 15,1 & 17,3 \\
& Gavinha & 43,7 & 37,7 & 40,4 \\
\hline
\end{tabular}

Tabela 3 - Matriz de similaridade florística (Jaccard) das espécies de trepadeiras amostradas nas florestas semideciduais em Uberlândia, MG. FSJ= Fazenda São José; FEG= Fazenda Experimental do Glória; $\mathrm{FAI}=$ Fazenda Irara.

Table 3 - Floristic similarity matrix (Jaccard) of the climbing species sampled in semideciduous forests in Uberlândia, MG, Brazil. FSJ= Fazenda São José; FEG= Fazenda Experimental do Glória; FAI= Fazenda Irara.

\begin{tabular}{lccc}
\hline & FSJ & FEG & FAI \\
\hline FSJ & 1 & & \\
FEG & 0,42 & 1 & \\
FAI & 0,38 & 0,33 & 1 \\
\hline
\end{tabular}

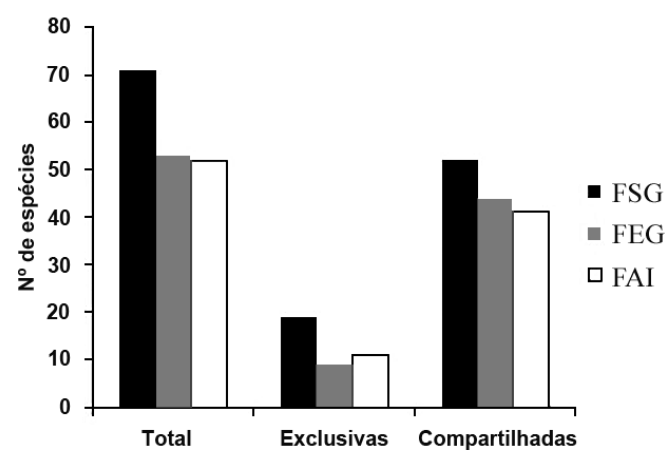

Figura 2 - Número de espécies de trepadeiras exclusivas a um dado fragmento ou compartilhada por dois ou mais fragmentos de floresta semidecidual. FEG = Fazenda Experimental do Glória, FSJ = Fazenda São José, FAI = Fazenda Irara.

Figure 2 - Number of species that were exclusively found in one fragment or that was shared between two or more fragments. FEG $=$ Fazenda Experimental do Glória, FSJ = Fazenda São José, FAI = Fazenda Irara.

As famílias com maior número de espécies nos fragmentos de Uberlândia, Bignoniaceae, Sapindaceae e Malpighiaceae, estão incluídas entre as mais ricas listadas por Gentry (1991) para florestas neotropicais. Resultados semelhantes também foram encontrados em outros trabalhos em florestas semideciduais no estado de São Paulo desenvolvidos por Hora \& Soares (2002), Tibiriçá et al. (2006) e Udulutsch et al. (2010). Estas três famílias são constituídas principalmente por gêneros de lianas (Tibiriçá et al. 2006). Bignoniaceae possui a tribo Bignonieae com predominância de trepadeiras lenhosas (Gentry 1991; Lohmann 2006) podendo ser uma explicação de sua maior ocorrência.

Semelhante ao presente estudo os modos de escalada volúvel e gavinha foram os mais 
encontrados nas amostragens em fragmentos de florestas semideciduais segundo Santos et al. (2009). Estudos desenvolvidos por Gentry (1991) mostraram que, em regiões neotropicais, a estratégia gavinha é a mais especializada, além de ser restrita a poucas famílias com grande número de espécies. O menor número de espécies com o modo de escalada apoiante aqui encontradas pode ser associado com o menor grau de especialização dessas espécies (Gentry 1991).

Quanto ao hábito encontramos cerca de $2 / 3$ de espécies lenhosas estando de acordo com a estimativa encontrada por Udulutsch et al. (2004), Durigon et al. (2009) em florestas semideciduais. Esse resultado opõe ao que foi registrado por Gentry (1991), no qual trepadeiras lenhosas e herbáceas têm a mesma contribuição em número de espécies em florestas tropicais de baixa altitude. Como são poucos os levantamentos florísticos neste tipo de fisionomia que classificam as trepadeiras e ou lianas segundo o hábito (Morellato \& Leitão Filho 1998; Udulutsch et al. 2004; Durigon et al. 2009), pode-se inferir pouco sobre esta variação das formas lenhosas e herbáceas.

Assim como outras características ecológicas das trepadeiras aqui já mencionadas, a dispersão anemocórica, mais encontrada entre os três fragmentos, esteve relacionada com as famílias mais abundantes em espécies (Bignoniaceae, Sapindaceae, Malpighiaceae). Esta forma de dispersão associa-se aos ambientes de sazonalidade climática (Howe \& Smallwood 1982), que facilita a anemocoria (Mantovani \& Martins 1988). Nos fragmentos estudados também está relacionada com as trepadeiras lenhosas que de acordo com Morellato \& Leitão Filho (1996), maturam seus frutos durante a estação relativamente seca, quando as árvores estão com menor percentual de folhas (Mantovani \& Martin 1988).

Em relação à similaridade florística entre fragmentos, Kent \& Coker (1992) consideram alta similaridade valores acima de 50\%. Apesar de estarem localizados no mesmo município e com menos de $35 \mathrm{~km}$ de distância um do outro, os três fragmentos florestais tiveram baixa similaridade florística. Isto já foi observado em outros trabalhos com trepadeiras, tais como Santos et al. (2009) explicando a alta diversidade $\beta$, ou seja, a composição de espécies bastante heterogênea entre as áreas.

Portanto, o presente estudo reafirmou a importância das trepadeiras na composição florística de florestas semideciduais conferindo a essas, elevada heterogeneidade taxonômica. Com isso, destacamos a necessidade de mais estudos destas plantas em áreas de floresta semidecidual no estado de Minas Gerais e no Brasil, principalmente aqueles relacionados a fatores que possam influenciar na composição florística de cada fragmento, tais como: umidade e fertilidade do solo, intensidade luminosa e disponibilidade de estruturas de apoio.

\section{Agradecimentos}

Agradecemos aos pesquisadores Eric Koiti Okyama Hattori (Asteraceae) e Maria Silvia Ferrucci (Sapindaceae), que auxiliaram na identificação das espécies; aos curadores dos herbários da Universidade de Campinas e Herbário Escola Superior de Agricultura Luiz de Queiroz, que disponibilizaram a consulta ao acervo; ao órgão financiador Fundação de Amparo à Pesquisa do Estado de Minas Gerais- FAPEMIG, o auxílio para a realização desta pesquisa; e a Pedro Braunger de Vasconcelos, o tratamento do mapa com a localização das áreas de estudo.

\section{Referências}

Bernacci, L.C. \& Leitão Filho, H.F. 1996. Flora fanerogâmica da floresta da Fazenda São Vicente, Campinas, SP. Revista Brasileira de Botânica 19: 149-164.

Bremer, B.; Bremer, K.; Chase, M.W.; Fay, M.F.; Reveal, J.L.; Soltis, D.E. \& Stevens, P.F. 2009. An update of the Angiosperm Phylogeny Group classification for the orders and families of flowering plants: APG III. Botanical Journal of the Linnean Society 161: 105-121.

DeWalt, S.J.; Schnitzer, S.A. \& Denslow, J.S. 2000. Density and diversity of lianas along a chronosequence in a central Panamanian lowland forest. Journal of Tropical Ecology 16: 1-19.

DeWalt, S.J.; Schinitzer, S.A.; Chave, J.; Bonger, F.; Burnham, R.J.; Cai, Z.; Chuyong, G.; Clarck, D.B.; Ewaango, C.E.N.; Gerwing, J.J.; Gortaire, E.; Hart, T.; Ibarra-Manríques, G.; Ickes, K.; Kenfack, D.; Macía, M.J.; Makana, J.; Martínez-Ramos, M.; Mascaro, J.; Moses, S.; Muller-Landau, H.C.; Parren, M.P.E.; Parthasarathy, N.; Pérez-Salicrup, D.R.; Putz, F.E.; Romero-Saltos, H. \& Thomas, D. 2010. Annual rainfall and seasonality predict pantropical patterns of liana density and basal area. Biotropica 42: 309-317.

Durigon, J.; Canto-Dorow, T.S. \& Eisinger, S.M. 2009. Composição florística de trepadeiras ocorrentes em bordas de fragmentos de floresta estacional, Santa Maria, Rio Grande do Sul, Brasil. Rodriguésia 60: 415-422. 
Emmons, L.H. \& Gentry, A.H. 1983. Tropical forest structure and the distribution of gliding and prehensile-tailed vertebrates. The American Naturalist 12: 513-524.

Fonseca, R.C.B. \& Oliveira, R.E. 1998. Ecologia de lianas e manejo de fragmentos florestais. Série Botânica IPEF 12: 43-64.

Gentry, A.H. 1985. An ecotaxonomic survey of panamanain lianas. In: Arcy, W.G.D. \& Correa, M. (eds.). Historia natural de Panama. Vol. 10. Missouri Botanical Garden, St. Louis. Pp. 29-42.

Gentry, A.H. 1991. The distribution and evolution of climbing plants. In: Putz, F.E. \& Mooney, H.A. (eds.). The biology of vines. Cambridge University Press, Cambridge. Pp. 3-49.

Granados, J. \& Korner, C. 2002. In deep shade, elevated $\mathrm{CO}_{2}$ increases the vigor of tropical climbing plants. Global Change Biology 8: 1-9.

Haridasan, M. \& Araújo, G.M. 2005. Perfil nutricional de espécies lenhosas de duas florestas semidecíduas em Uberlândia, MG. Revista Brasileira de Botânica 28: 295-303.

Hergaty, E.E. 1991. Vine-host interactions. In: Putz, F.E. \& Mooney, H.A. The biology of vines. Cambridge University Press, Cambridge. Pp. 357-375.

Hergaty, E.E. \& Caballé, G. 1991. Distributios and abundance of vines in forest communities. In: Putz, F.E. \& Mooney, H.A. The biology of vines. Cambridge University Press, Cambridge. Pp. 313-335.

Hora, R.C. \& Soares, J.J. 2002. Estrutura fitossociológica da comunidade de lianas em uma floresta estacional na Fazenda Cachim, São Carlos, SP. Revista Brasileira de Botânica 25: 323-329.

Howe, H.F. \& Smallwood, J. 1982. Ecology of seed dispersal. Annual Review of Ecology and Systematic 13: 201-228.

Isnard, S. \& Silk, W.K. 2009. Moving with climbing plants from Charles Darwin's time into the $21^{\text {st }}$ century. American Journal of Botany 96: 1205-1221.

Kent, M. \& Coker, P. 1992. Vegetations descriptions and analysis. Belhaven Press, London. 384p.

Klink, C.A. \& Machado, R.B. 2005. A conservação do Cerrado brasileiro. Megadiversidade 1: 147-155.

Köppen, W.P. 1948. Climatologia. Fondo de Cultura Económica, Ciudad de México, Buenos Aires. $479 p$.

Laurence, W.F.; Peréz-Salicrup, D.; Dalemônica, P.; Fearnside, P.M.; D’angelo, S.; Jerozolinski, A.; Pohl, L. \& Levejoy, T.E. 2001. Rain forest fragmentation and the structure of Amazonian liana communities. Ecology 82: 105-116.

Lohmann, L. 2006. Untangling the phylogeny of Neotropical lianas (Bignonieae, Bignoniaceae). American Journal of Botany 93: 304-318.
Lopes, S.F. 2010. Padrões florísticos e estruturas das florestas estacionais semideciduais do Triângulo Mineiro, MG. Tese de Doutorado. Universidade Federal de Uberlândia, Uberlândia.192p.

Lopes, S.F.; Schiavini, I.; Oliveira, A.P. \& Vale, V.S. 2012. An ecological comparison of floristic composition in seasonal semideciduous forest in Southeast Brazil: implications for conservation. International Journal of Forestry Research 2012: 1-14.

Mantovani, W. \& Martins, F.R. 1988. Variações fenológicas das espécies do cerrado da Reserva Biológica de Moji Guaçu, estado de São Paulo. Revista Brasileira de Botânica 11: 101-112.

Morellato, P.C. \& Leitão Filho, H.F. 1996. Reproductive phenology of climbers is Southeastern Brazilian forest. Biotropica 28: 180-191.

Morellato, P.C. \& Leitão Filho, H.F. 1998. Levantamento florístico da comunidade de trepadeiras de uma floresta semidecídua no Sudeste do Brasil. Boletim do Museu Nacional, Nova Série Botânica 103: 1-15.

Mueller-Dombois, D. \& Ellenberg, H. 1974. H. Aims and methods of vegetation ecology. Willey and Sons, New York. 547p.

Phillips, O.L.; Vásquez, M.R.; Arroyo, L.; Baker, T.; Killeen, T.; Lewis, S.L.; Yandvinder, M.; Mendonza,A.M.; Neil, D.; Vargas, P.C.; Alexiades, M.; Cerón, C.; Di Fiore, A.; Erwin, T.; Jardim, A.; Palacios, W.; Saldias, M.; Vinceti, B. 2002. Increasing dominance of large lianas in Amazonian forests. Nature 418: 770-774.

Phillips, O.L.; Vásquez Martínez, R.; Monteagudo Mendoza, A.; Baker, T.R. \& Núnêz Vargas, P. 2005. Large lianas as hyperdynamic elements of the tropical forest canopy. Ecology 86: 1250-1258.

Putz, F.E. 1984. The natural history of lianas on Barro Colorado Island, Panama. Ecology 65: 1713-1724.

Putz, F.E. \& Chai, P. 1987. Ecological studies of lianas in Lambir National Park, Sarawak. Journal of Ecology 75: 523-531.

Putz, F. E. 2005. Ecologia das trepadeiras. Ecology. Info 24: 1-15.

Rezende, A.A. \& Ranga, N.T. 2005. Lianas da Estação Ecológica do Noroeste Paulista, São José do Rio Preto/Mirassol, SP, Brasil. Acta Botanica Brasilica 19: 273-279.

Rezende, A.A.; Ranga, N.T. \& Pereira, R.A.S. 2007. Lianas de uma floresta estacional semidecidual, município de Paulo de Faria, norte do estado de São Paulo, Brasil. Revista Brasileira de Botânica 30: 451-461.

Santos, K.; Kinoshita, L.S. \& Rezende, A.A. 2009. Species composition of climbers in seasonal semideciduous forest fragments of Southeastern Brazil. Biota Neotropica 9: 175-188.

Schnitzer, S.A. \& Carson, W.P. 2001. Treefall gaps and the maintenance of species diversity in a tropical forest. Ecology 82: 913-919. 
Schnitzer, S.A. \& Bongers, F. 2002. The ecology of lianas and their role in forest. Trends in Ecology \& Evolution 17: 223-230.

Schnitzer, S.A. 2005. A mechanistic explanation for global patterns of liana abundance and distribution. The American Naturalist 166: 262-276.

Sfair, J.C. \& Martins, F.R. 2011. The role of the heterogeneity on climber diversity: is liana diversity related to tree diversity? Global Journal of Biodiversity Science and Management 1: 1-10.

Silva, M.I.S.; Guimarães, E.C. \& Tavares, M. 2008. Previsão da temperatura média mensal de Uberlândia, MG, com modelos de séries temporais. Revista Brasileira de Engenharia Agrícola e Ambiental 12: 480-485.

Tabarelli, M. \& Gascon, C. 2005. Lessons from fragmentation research: improving management and policy guidelines for biodiversity conservation. Conservation Biology 19: 734-739.
Tibiriçá, Y.J.A.; Coelho, L.F.M. \& Moura, L.C. 2006. Florística de lianas em um fragmento de floresta estacional semidecidual, Parque Estadual de Vassununga, Santa Rita do Passa Quatro, SP, Brasil. Acta Botanica Brasilica 20: 339-346.

Udulutsch, R.G.; Assis, M.A. \& Picchi, D.G. 2004. Florística de trepadeiras numa floresta estacional semidecídua, Rio Claro-Araras, estado de São Paulo, Brasil. Revista Brasileira de Botânica 27: 125-134.

Udulutsch, R.G.; Souza, V.C.; Rodrigues, R.R. \& Dias, P. 2010. Composição florística e chaves de identificação para as lianas da Estação Ecológica dos Caetetus, estado de São Paulo, Brasil. Rodriguésia 61: 715-730.

Van Der Pijil, L. 1982. Principles of dispersal in higher plants. $2^{\text {nd }}$ ed. Springer Verlag, New York. 211p.

Villagra, B.L.P. \& Neto, S.R. 2010. Florística de trepadeiras no Parque Estadual das Fontes do Ipiranga, São Paulo, SP, Brasil. Revista Brasileira de Biociências 8: 186-200. 